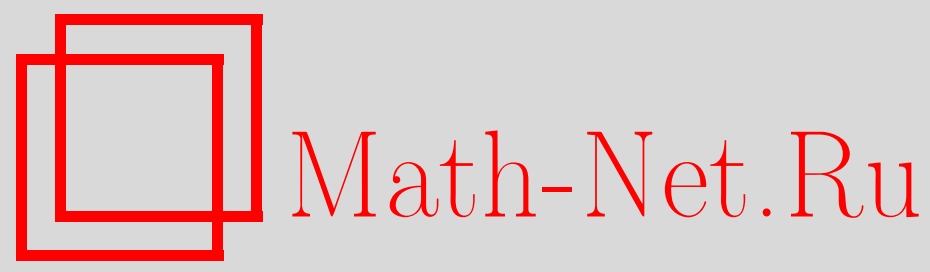

В. П. Маслов, Новое представление оператора Гамильтона для бозонов и фермионов. Квантование свободной энергии и зависимость критерия Ландау от температуры, Матем. заметки, 2000, том 68, выпуск 6, 945-947

DOI: https://doi.org/10.4213/mzm1019

Использование Общероссийского математического портала Math-Net.Ru подразумевает, что вы прочитали и согласны с пользовательским соглашением http://www . mathnet.ru/rus/agreement

Параметры загрузки:

IP : 52.87 .193 .239

26 апреля 2023 г., 18:00:56

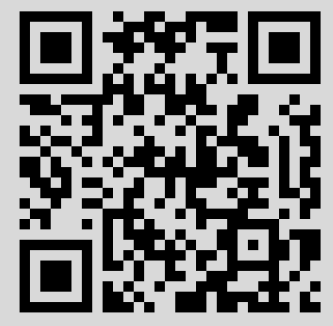




\section{НОВОЕ ПРЕДСТАВЛЕНИЕ ОПЕРАТОРА ГАМИЛЬТОНА ДЛЯ БОЗОНОВ И ФЕРМИОНОВ. КВАНТОВАНИЕ СВОБОДНОЙ ЭНЕРГИИ И ЗАВИСИМОСТЬ КРИТЕРИЯ ЛАНДАУ ОТ ТЕМПЕРАТУРЫ}

\section{В.П. Маслов}

Рассмотрим систему $N$ тождественных частиц на трехмерном торе $\mathbb{T}^{3}$, длина сторон которого $L$. Пусть гамильтониан системы имеет вид

$$
\widehat{H}_{N}=-\frac{\hbar^{2}}{2 m} \sum_{j=1}^{N} \Delta_{j}+U_{0} \sum_{1 \leqslant j<k \leqslant N} v\left(\frac{x_{j}-x_{k}}{a}\right)
$$

где $x_{j} \in \mathbb{T}^{3}, \Delta$ - оператор Лапласа, $m$ - масса частиц, $\hbar$ - постоянная Планка, $U_{0}, a$ - заданные постоянные, $v(x)$ - непрерьвная четная функция на торе. Если частицы являются бозонами или фермионами, гамильтониану (1) отвечает вторично квантованный [1] оператор

$$
\widehat{H}=-\frac{\hbar^{2}}{2 m} \int d x \widehat{\psi}^{+}(x) \Delta \widehat{\psi}^{-}(x)+U_{0} \iint d x d y v\left(\frac{x_{j}-x_{k}}{a}\right) \widehat{\psi}^{+}(x) \widehat{\psi}^{+}(y) \widehat{\psi}^{-}(y) \widehat{\psi}^{-}(x)
$$

где операторы $\widehat{\psi}^{ \pm}(x)$ являются операторами рождения и уничтожения частиц соответственно в бозонном $\mathscr{H}_{B}$ или в фермионном $\mathscr{H}_{F}$ пространствах Фока [1]. Пусть $k>0$ - целое число. Введем операторнозначный функционал

$$
\begin{aligned}
\widehat{\rho}= & \sum_{N_{1}=0}^{\infty} \ldots \sum_{N_{k}=0}^{\infty} \frac{1}{N_{1} ! \cdots N_{k} !} \prod_{l=1}^{k}\left(\sum_{j^{l}=0}^{\infty} \int \cdots \int d x_{1}^{l} d y_{1}^{l} \cdots d x_{l}^{l} d y_{l}^{l} b_{l}\left(x_{1}^{l}, \ldots, x_{l}^{l}, j^{l}\right){\stackrel{2}{\psi^{+}}}^{+}\left(x_{1}^{l}\right) \ldots\right. \\
& \left.\times \widehat{\psi}^{+}\left(x_{l}^{l}\right) b_{l}^{*}\left(y_{1}^{l}, \ldots, y_{l}^{l}, j^{l}\right) \widehat{\psi}^{-}\left(y_{1}^{l}\right) \cdots \widehat{\psi}^{-}\left(y_{l}^{l}\right)\right)^{N_{l}} \exp \left(-\int d z \widehat{\psi}^{+}(z) \widehat{\psi}^{-}(z)\right)
\end{aligned}
$$

который определен на наборах из $k$ функций $b_{l}\left(x_{1}^{l}, \ldots, x_{l}^{l}, j\right), l=1, \ldots, k$, таких, что

$$
\sum_{l=1}^{k} \sum_{j=0}^{\infty} \int \cdots \int d x_{1} \cdots d x_{l}\left|b_{l}\left(x_{1}^{l}, \ldots, x_{l}^{l}, j\right)\right|^{2}<\infty
$$

и значения которого есть операторы в пространстве $\mathscr{H}_{B}$. Числа над операторами в (2) обозначают порядок их действия [2]. Введем также аналогичный функционал, принимающий значения в пространстве $\mathscr{H}_{F}$ :

$$
\begin{aligned}
\widehat{\rho}= & \sum_{N_{1}=0}^{\infty} \cdots \sum_{N_{k}=0}^{\infty} \frac{1}{N_{1} ! \cdots N_{k} !} \sum_{j_{1}^{1}=0}^{\infty} \cdots \sum_{j_{N_{1}}^{1}=0}^{\infty} \cdots \sum_{j_{1}^{k}=0}^{\infty} \cdots \sum_{j_{N_{k}}^{k}=0}^{\infty} \int \cdots \int d x_{1}^{1} d y_{1}^{1} \cdots d x_{N_{1}}^{1} \\
& \times d y_{N_{1}}^{1} \cdots d x_{1}^{k} d y_{1}^{k} \cdots d x_{k N_{k}}^{k} d y_{k N_{k}}^{k} \widehat{\psi}^{+}\left(x_{1}^{1}\right) \cdots \widehat{\psi}^{+}\left(x_{N_{1}}^{1}\right) \cdots \widehat{\psi}^{+}\left(x_{1}^{k}\right) \cdots \widehat{\psi}^{+}\left(x_{k N_{k}}^{k}\right) \widehat{P}_{0} \\
& \times b_{1}\left(x_{1}^{1}, j_{1}^{1}\right) \cdots b_{1}\left(x_{N_{1}}^{1}, j_{N_{1}}^{1}\right) \cdots b_{k}\left(x_{1}^{k}, \ldots, x_{k}^{k}, j_{1}^{k}\right) \cdots b_{k}\left(x_{k N_{k}-k+1}^{k}, \ldots, x_{k N_{k}}^{k}, j_{N_{k}}^{k}\right) \\
& \times b_{1}^{*}\left(y_{1}^{1}, j_{1}^{1}\right) \cdots b_{1}^{*}\left(y_{N_{1}}^{1}, j_{N_{1}}^{1}\right) \cdots b_{k}^{*}\left(y_{1}^{k}, \ldots, y_{k}^{k}, j_{1}^{k}\right) \cdots b_{k}^{*}\left(y_{k N_{k}-k+1}^{k}, \ldots, y_{k N_{k}}^{k}, j_{N_{k}}^{k}\right) \\
& \times \widehat{\psi}^{-}\left(y_{k N_{k}}^{k}\right) \cdots \widehat{\psi}^{-}\left(y_{1}^{k}\right) \cdots \widehat{\psi}^{-}\left(y_{N_{1}}^{1}\right) \cdots \widehat{\psi}^{-}\left(x_{1}^{1}\right),
\end{aligned}
$$


где $\widehat{P}_{0}$ - оператор проектирования на вакуумный вектор [1] пространства $\mathscr{H}_{F}$. Функциональные аргументы у функционалов $\widehat{\rho}$, определенных формулами (2) и (3), будем опускать. Введем далее для бозонов и фермионов функционал

$$
\begin{aligned}
\mathscr{H} & {\left[b_{1}^{*}\left(x_{1}^{1}, j^{1}\right), b_{1}\left(x_{1}^{1}, j^{1}\right), \ldots, b_{k}^{*}\left(x_{1}^{k}, \ldots, x_{k}^{k}, j^{k}\right), b_{k}\left(x_{1}^{k}, \ldots, x_{k}^{k}, j^{k}\right)\right] } \\
& =\operatorname{Sp}(\widehat{\rho} \widehat{H}) \exp \left(-\sum_{l=1}^{k} \sum_{j=0}^{\infty} \int \cdots \int d y_{1} \cdots d y_{k} b_{l}^{*}\left(y_{1}, \ldots, y_{l}, j\right) b_{l}\left(y_{1}, \ldots, y_{k}, j\right)\right),
\end{aligned}
$$

где в бозонном случае $\widehat{\rho}$ имеет вид (2), а в фермионном - (3).

В работах [3]-[5] были введены пространства $\mathscr{F} N_{1}, \ldots, N_{k}$ и их бозонные и фермионные подпространства $\mathscr{F}_{N_{1}, \ldots, N_{k}}^{B}$ и $\mathscr{F}_{N_{1}, \ldots, N_{k}}^{F}$, а также пространства $\mathscr{F}=\bigoplus_{N_{1}, \ldots, N_{k}=0}^{\infty} \mathscr{F}_{N_{1}, \ldots, N_{k}}$ для $k$ типов кластеров. Пространства $\mathscr{F}$ являются пространствами Фока, и в них определены операторы рождения и уничтожения $\widehat{b}_{l}^{ \pm}\left(x_{1}, \ldots, x_{l}, j\right), l=1, \ldots, k$. Кроме того, в $\mathscr{F}$ вводятся [3]-[5] осредненные гамильтонианы следуюшим образом:

$$
\overline{\widehat{\mathscr{H}}}=\mathscr{H}\left[\widehat{b}_{1}^{+}\left(x_{1}^{1}, j^{1}\right), \widehat{b}_{1}^{-}\left(x_{1}^{1}, j^{1}\right), \ldots, \widehat{b}_{k}^{+}\left(x_{1}^{k}, \ldots, x_{k}^{k}, j^{k}\right), \widehat{b}_{k}^{-}\left(x_{1}^{k}, \ldots, x_{k}^{k}, j^{k}\right)\right] .
$$

\section{Имеет место следующая}

ТЕорема. Проекиия осредненного гамильтониана (4) на подпространство $\mathscr{F}_{N_{1}, \ldots, N_{k}}^{B}$ в случае, когда $\widehat{\rho}$ имеет вид (2), и его проекиия на $\mathscr{F}_{N_{1}, \ldots, N_{k}}^{F}$ в случае, когда $\widehat{\rho}$ имеет вид (3), совпадает с гамильтонианом (1).

Рассмотрим теперь функционал

$$
\begin{aligned}
S & {\left[b_{1}^{*}\left(x_{1}^{1}, j^{1}\right), b_{1}\left(x_{1}^{1}, j^{1}\right), \ldots, b_{k}^{*}\left(x_{1}^{k}, \ldots, x_{k}^{k}, j^{k}\right), b_{k}\left(x_{1}^{k}, \ldots, x_{k}^{k}, j^{k}\right)\right] } \\
& =\operatorname{Reg} \operatorname{Sp}\left(\widehat{\rho} \ln \left(\frac{\widehat{\rho}}{\operatorname{Sp} \widehat{\rho}}\right)\right) \exp \left(-\sum_{l=1}^{k} \sum_{j=0}^{\infty} \int \cdots \int d y_{1} \cdots d y_{k} b_{l}^{*}\left(y_{1}, \ldots, y_{l}, j\right) b_{l}\left(y_{1}, \ldots, y_{k}, j\right)\right)_{(5)}
\end{aligned}
$$

Оператор

$$
\overline{\widehat{S}}=S\left[\widehat{b}_{1}^{+}\left(x_{1}^{1}, j^{1}\right), \widehat{b}_{1}^{-}\left(x_{1}^{1}, j^{1}\right), \ldots, \widehat{b}_{k}^{+}\left(x_{1}^{k}, \ldots, x_{k}^{k}, j^{k}\right), \widehat{b}_{k}^{-}\left(x_{1}^{k}, \ldots, x_{k}^{k}, j^{k}\right)\right]
$$

был введен в [3]-[5] и называется оператором әнтропии системы бозонов или фермионов при соответствующем выборе $\widehat{\rho}$ в виде (2) или (3). Числа $\lambda$, для которых

$$
(\widehat{\widehat{\mathscr{H}}}+\theta \overline{\widehat{S}}) \Phi=\lambda \Phi, \quad \Phi \in \mathscr{F}, \quad \Phi \neq 0
$$

назьваются [3]-[5] в соответствующем случае квантовыми значениями свободной энерәии системы бозонов или фермионов при температуре $\theta$.

В случае, когда взаимодействие между частицами мало, некоторые из квантовых значений свободной энергии определяются точками экстремума функционала $F=(\mathscr{H}+\theta S) / \operatorname{Sp} \widehat{\rho}$, где $\mathscr{H}$ и $S$ имеют вид (4) и (5), и системой уравнений в вариациях [6] около этих точек экстремума. В частности, для системы бозонов при $k=1$ в термодинамическом пределе при $L \rightarrow \infty, N \rightarrow \infty$, $N / L^{3} \rightarrow$ const существуют точки экстремума функционала $F$ следующего вида:

$$
b_{1}^{p_{0}}(x, j)=\frac{\sqrt{n_{q_{j}}-p_{0}}}{L^{3 / 2}} \exp \left(\frac{i q_{j} x}{a}\right)
$$


где $q_{j}=\left(l_{j}^{1}, l_{j}^{2}, l_{j}^{3}\right) 2 \pi a / L,\left(l_{j}^{1}, l_{j}^{2}, l_{j}^{3}\right)$ - взаимооднозначное отображение множества $j=0,1, \ldots$ на $\mathbb{Z}^{3}, p_{0}=\left(m^{1}, m^{2}, m^{3}\right) 2 \pi a / L, m^{1}, m^{2}, m^{3} \in \mathbb{Z}$ - параметр, нумерующий разные точки экстремума, $n_{p}$ являются решением системы уравнений

$$
\begin{aligned}
\varepsilon_{p} & +\frac{U_{0} a^{3} n_{0}}{L^{3}} \sum_{p^{\prime}}\left(\widetilde{v}(0)+\widetilde{v}\left(p-p^{\prime}\right)\right) n_{p^{\prime}}+\theta \ln \left(\frac{n_{p}}{1+n_{p}}\right) \\
& -\mu-\delta_{p 0}\left(\frac{U_{0} a^{3} n_{0}}{L^{3}} \widetilde{v}(0)+\theta \ln \left(\frac{n_{0}}{1+n_{0}}\right)\right)=0, \quad N=\sum_{p} n_{p}
\end{aligned}
$$

где $\widetilde{v}(p)=\int d x v(x) \exp (i p x), \varepsilon_{p}=\hbar^{2} p^{2} / 2 m a^{2}, \delta_{p q}$ - символ Кронекера. При этом система уравнений в вариациях, которая отвечает экстремуму (6) и определяет его устойчивость, имеет вид

$$
\begin{aligned}
\omega F_{p q}= & \frac{1}{N\left(a_{p}-a_{q}\right)}\left\{\delta _ { p p _ { 0 } } \left[n_{0} \frac{\hbar^{2}}{2 m a^{2}}\left(q^{2}-p_{0}^{2}\right) F_{p_{0} q}+n_{0}^{2} \frac{U_{0} a^{3}}{L^{3}} \widetilde{v}\left(q-p_{0}\right) F_{p_{0} q}\right.\right. \\
& -n_{0}^{2} \frac{U_{0} a^{3}}{L^{3}} \widetilde{v}\left(q-p_{0}\right) F_{2 p_{0}-q p_{0}}+n_{0} \sum_{p^{\prime}} n_{p^{\prime}} \frac{U_{0} a^{3}}{L^{3}}\left(\widetilde{v}\left(p^{\prime}-q\right)-\widetilde{v}\left(p^{\prime}-p_{0}\right)\right) F_{p_{0} q} \\
& \left.+n_{0} \frac{U_{0} a^{3}}{L^{3}} \sum_{p^{\prime} q^{\prime}}\left(\widetilde{v}\left(p^{\prime}-q^{\prime}\right)-\widetilde{v}\left(p^{\prime}-p_{0}\right)\right)\left(n_{p^{\prime}}-n_{q^{\prime}}\right) F_{p^{\prime} q^{\prime}} \delta_{q+p^{\prime}, q^{\prime}}\right] \\
& +\delta_{q p_{0}}\left[n_{0} \frac{\hbar^{2}}{2 m a^{2}}\left(p^{2}-p_{0}^{2}\right) F_{p p_{0}}+n_{0}^{2} \frac{U_{0} a^{3}}{L^{3}} \widetilde{v}\left(p-p_{0}\right) F_{p p_{0}}\right. \\
& -n_{0}^{2} \frac{U_{0} a^{3}}{L^{3}} \widetilde{v}\left(p-p_{0}\right) F_{p_{0} 2 p_{0}-p}+n_{0} \frac{U_{0} a^{3}}{L^{3}} \sum_{p^{\prime}} n_{p^{\prime}}\left(\widetilde{v}\left(p^{\prime}-p\right)-\widetilde{v}\left(p^{\prime}-p_{0}\right)\right) F_{p p_{0}} \\
& \left.+n_{0} \frac{U_{0} a^{3}}{L^{3}} \sum_{p^{\prime} q^{\prime}}\left(\widetilde{v}\left(p^{\prime}-q^{\prime}\right)-\widetilde{v}\left(q^{\prime}-p_{0}\right)\right)\left(n_{p^{\prime}}-n_{q^{\prime}}\right) F_{p^{\prime} q^{\prime}} \delta_{p^{\prime} p+q^{\prime}}\right] \\
& +\left(\frac{\hbar^{2}}{2 m a^{2}}\left(n_{p}-n_{q}\right)\left(q^{2}-p^{2}\right)+n_{0} \frac{U_{0} a^{3}}{L^{3}}\left(\widetilde{v}\left(q-p_{0}\right)-\widetilde{v}\left(p-p_{0}\right)\right)\left(n_{p}-n_{q}\right)\right. \\
& \left.+\frac{U_{0} a^{3}}{L^{3}} \sum_{p^{\prime}}\left(\widetilde{v}\left(q-p^{\prime}\right)-\widetilde{v}\left(p-p^{\prime}\right)\right)\left(n_{p}-n_{q}\right) n_{p^{\prime}}\right) F_{p q} \\
& \left.+\frac{U_{0} a^{3}}{L^{3}} \sum_{p^{\prime} q^{\prime}}\left(\widetilde{v}(q-p)+\widetilde{v}\left(q-q^{\prime}\right)\right) \delta_{q+p^{\prime}, q^{\prime}+p}\left(n_{p^{\prime}}-n_{q^{\prime}}\right)\left(n_{p}-n_{q}\right) F_{p^{\prime} q^{\prime}}\right\}
\end{aligned}
$$

где $a_{p}=n_{p} /\left(1+n_{p}\right)$. При температуре $\theta$ и скорости $p_{0}$, для которых $n_{0} \sim N$ и все собственные значения $\omega$ системы (7) действительны, в бозонной системе существует конденсат и сверхтекучесть. При этом действительность $\omega$ является аналогом критерия Ландау исчезновения сверхтекучести. Исходя из этого, можно при фиксированной температуре $\theta$ определить критическую скорость, при которой нарушается действительность $\omega$ и исчезает сверхтекучесть.

\section{СПИСОК ЦИТИРОВАННОЙ ЛИТЕРАТУРЫ}

1. Березин Ф. А. Метод вторичного квантования. М.: Наука, 1986. 2. Маслов В. П. Операторные методы. М.: Наука, 1973. 3. Маслов В. П. // ТМФ. 2000. Т. 125. № 3. 4. Маслов В. П. // Функцион. анализ и его прилож. 1999. Т. 33. № 4. С. 50-64. 5. Маслов В. П. // Функцион. анализ и его прилож. 2000. Т. 34. № 4. С. 35-48. 6. Маслов В. П. Комплексный метод ВКБ в нелинейных уравнениях. М.: Наука, 1977. 